\title{
A Quenched Molecular Dynamics (QMD)-ROESY Study of a Series of Semi- biosynthetically Monoacylated Anthocyanins
}

Neil A. Whittemore ${ }^{\dagger, t}$, Karen T. Welch ${ }^{t,}$, James R. Cox ${ }^{\dagger,}$, Donald K. Dougall, David C. Baker ${ }^{\dagger} *$

Page S2 Figure S1. A plot of $\phi_{2} v s . \psi_{2}$ of 1,000 structures of 1 after 1-ns molecular dynamics at $600 \mathrm{~K}$.

Page S3 Figure S2. A plot of $\phi_{2} v s . \psi_{2}$ of 1,000 structures of 1 after minimization.

Page S4 Figure S3. A plot of $\phi_{3} v s . \psi_{3}$ of 1,000 structures of 1 after 1-ns molecular dynamics at $600 \mathrm{~K}$.

Page S5 Figure S4. A plot of $\phi_{3} v s . \psi_{3}$ of 1,000 structures of 1 after minimization.

Page S6 Figure S5. A plot of dihedral ${ }_{4} v s . \omega_{3}$ of 1,000 structures of 1 after 1-ns molecular dynamics at $600 \mathrm{~K}$.

Page S7 Figure S6. A plot of dihedral ${ }_{4} v s . \omega_{3}$ of 1,000 structures of 1 after minimization.

Page S8 Table S1. NMR-derived restraints of anthocyanin 2 in methanol- $d_{4}$.

Page S9 Figure S7. Conformational families of anthocyanin 2.

Page S10 Table S2. NMR-derived restraints of anthocyanin 3 in methanol- $d_{4}$.

Page S11

Page S12

Figure S8. Conformational families of anthocyanin 3.

Page S13

Page S14

Page S15

Table S3. NMR-derived restraints of anthocyanin 4 in methanol- $d_{4}$.

Figure S9. Some conformational families of anthocyanin 4.

Figure S10. The remaining conformational families of anthocyanin 4.

Table S4. NMR-derived restraints of anthocyanin 5 in methanol- $d_{4}$.

Page S16 Figure S11. Conformational families of anthocyanin 5. 


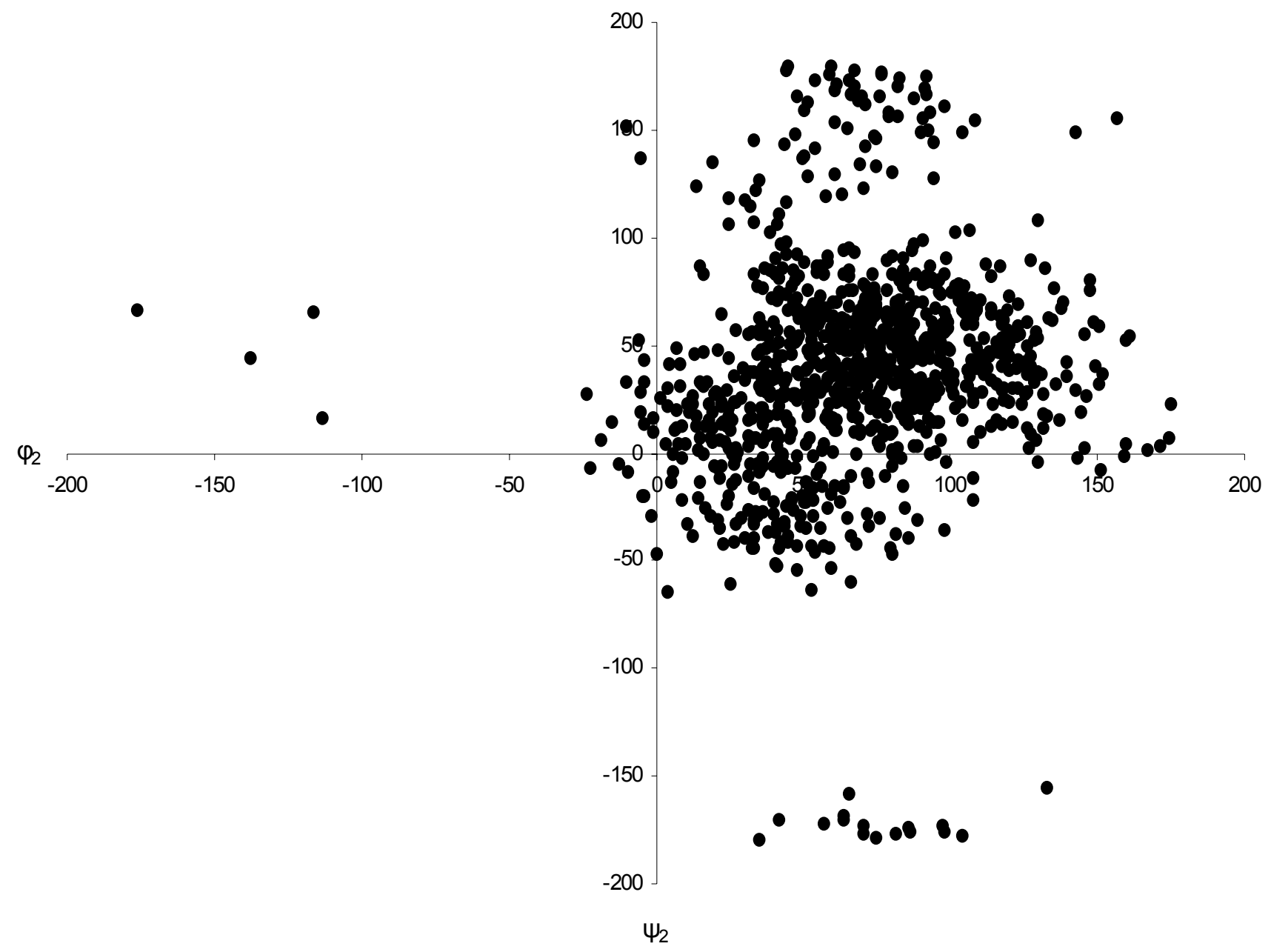

Figure S1. A plot of $\phi_{2} v s . \psi_{2}$ of 1,000 structures of 1 after 1-ns molecular dynamics at $600 \mathrm{~K}$. 


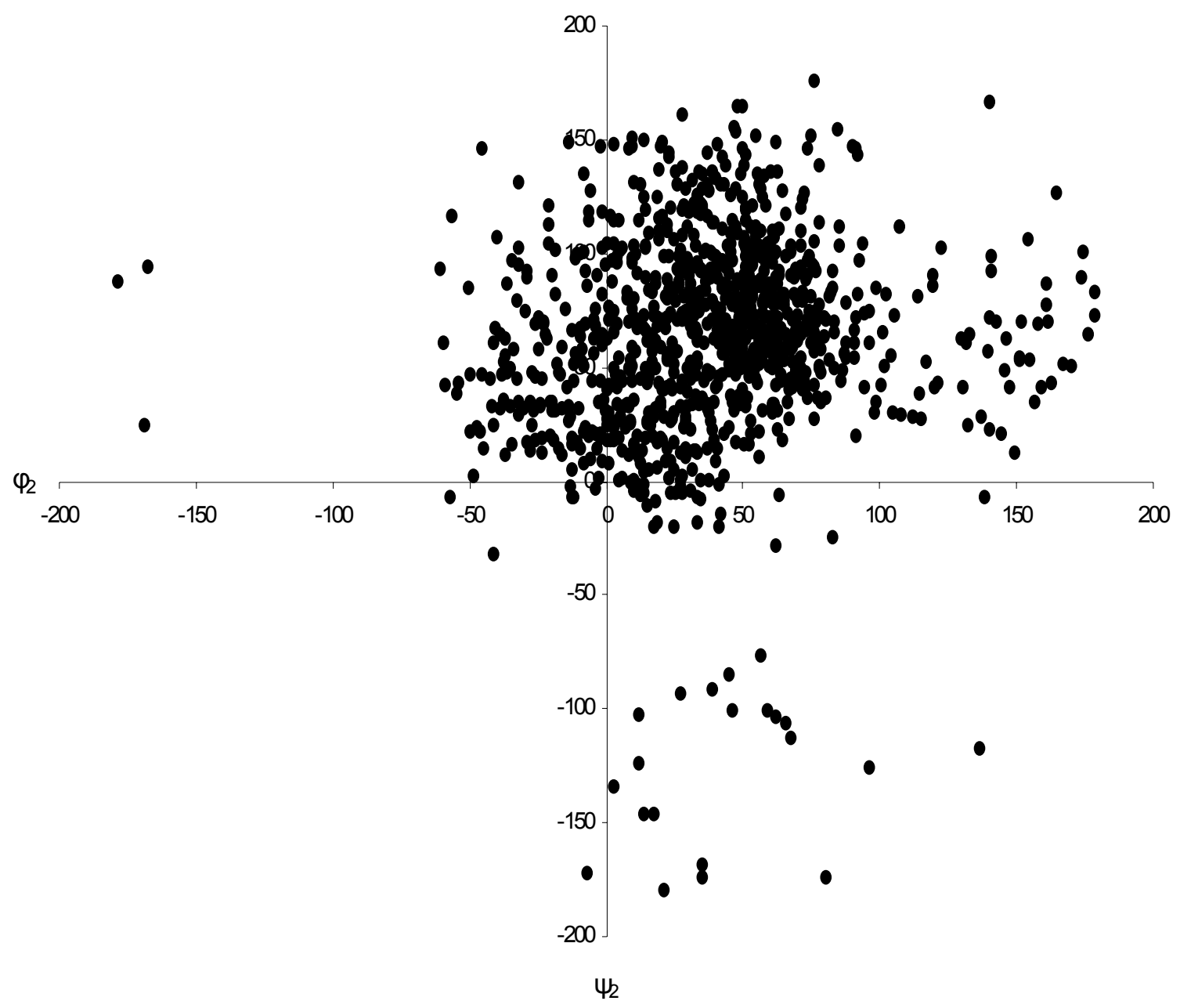

Figure S2. A plot of $\phi_{2} v s . \psi_{2}$ of 1,000 structures of 1 after minimization. 


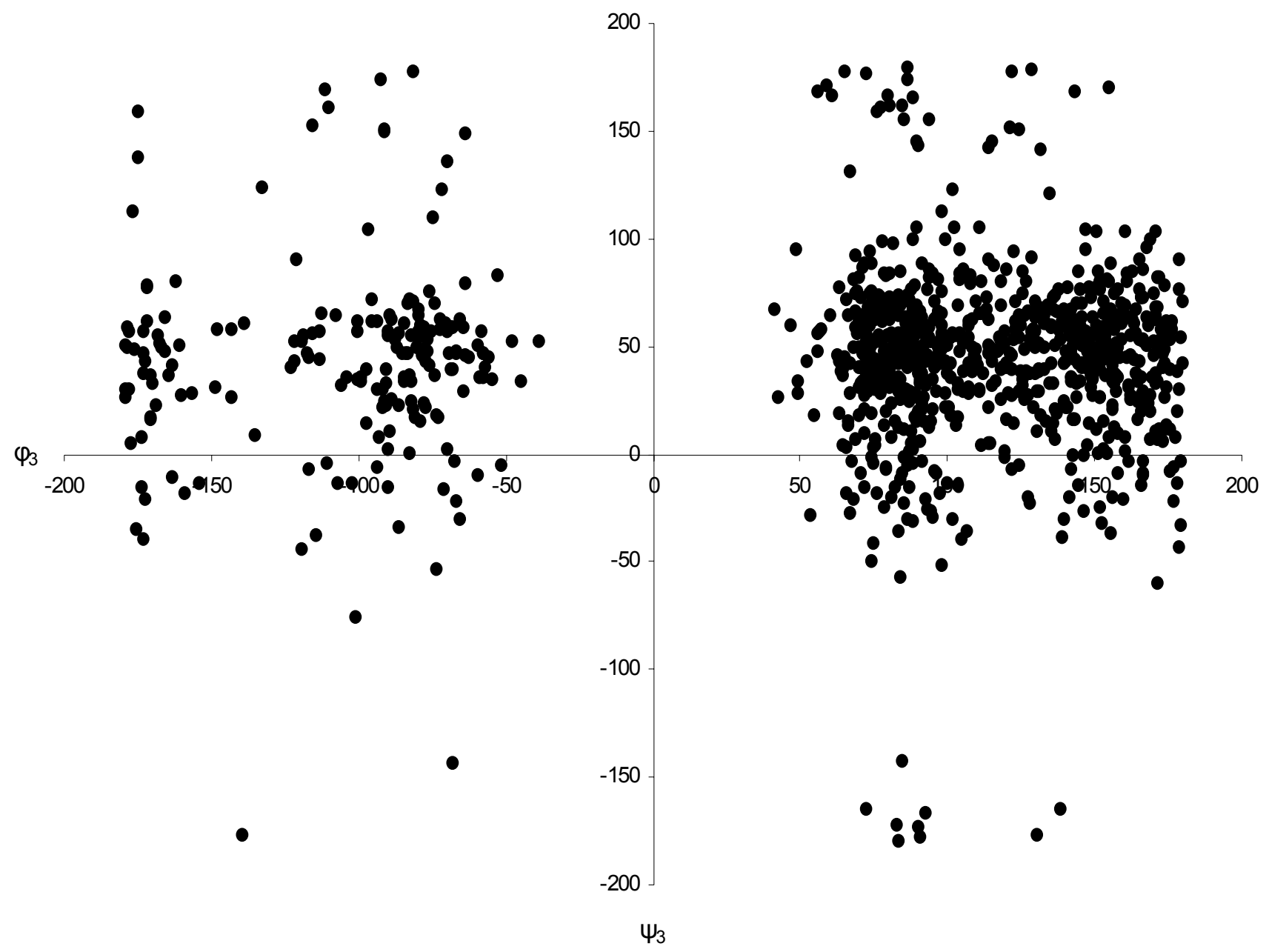

Figure S3. A plot of $\phi_{3} v s . \Psi_{3}$ of 1,000 structures of 1 after 1 -ns molecular dynamics at $600 \mathrm{~K}$. 


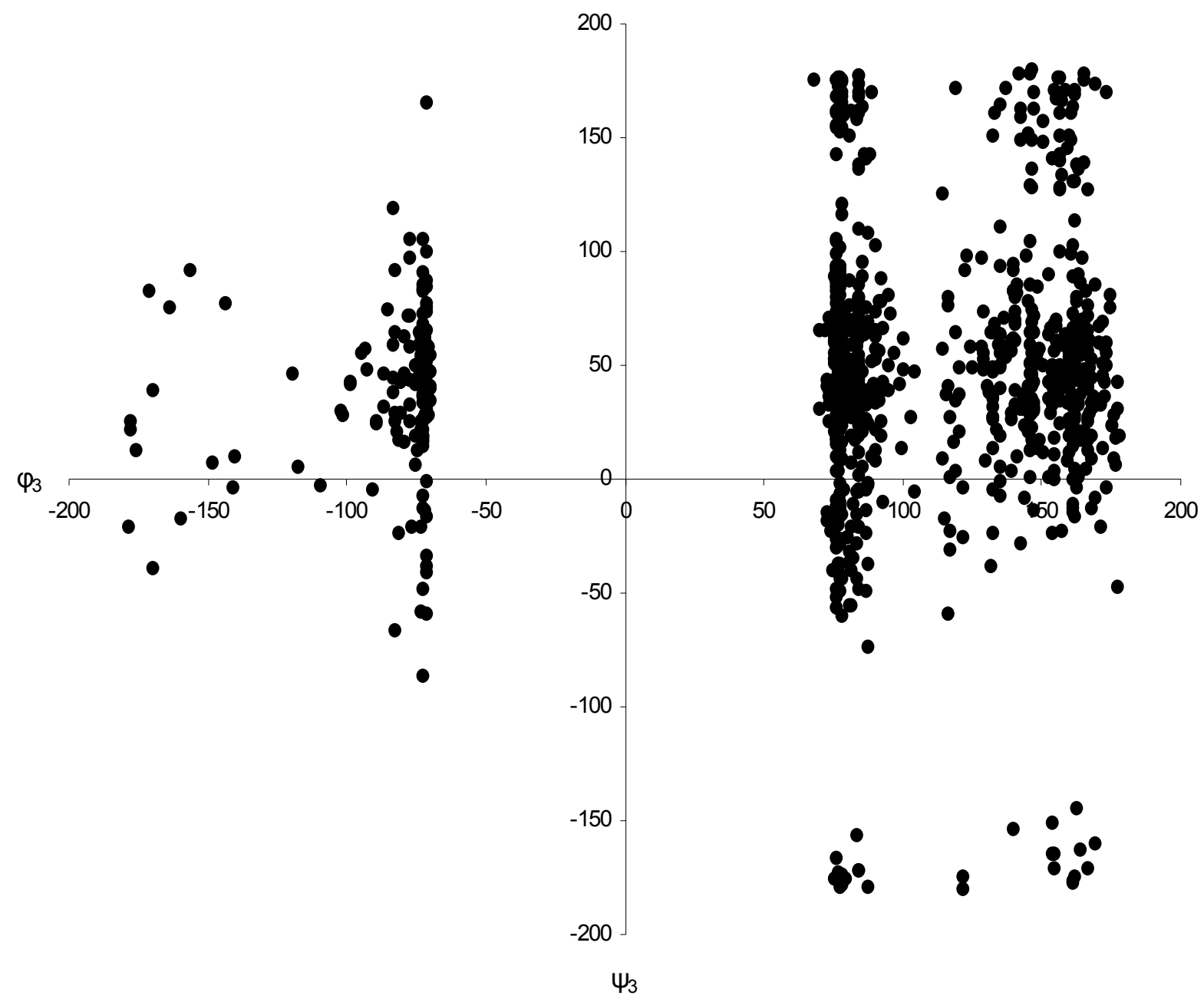

Figure S4. A plot of $\phi_{3} v s . \psi_{3}$ of 1,000 structures of 1 after minimization. 


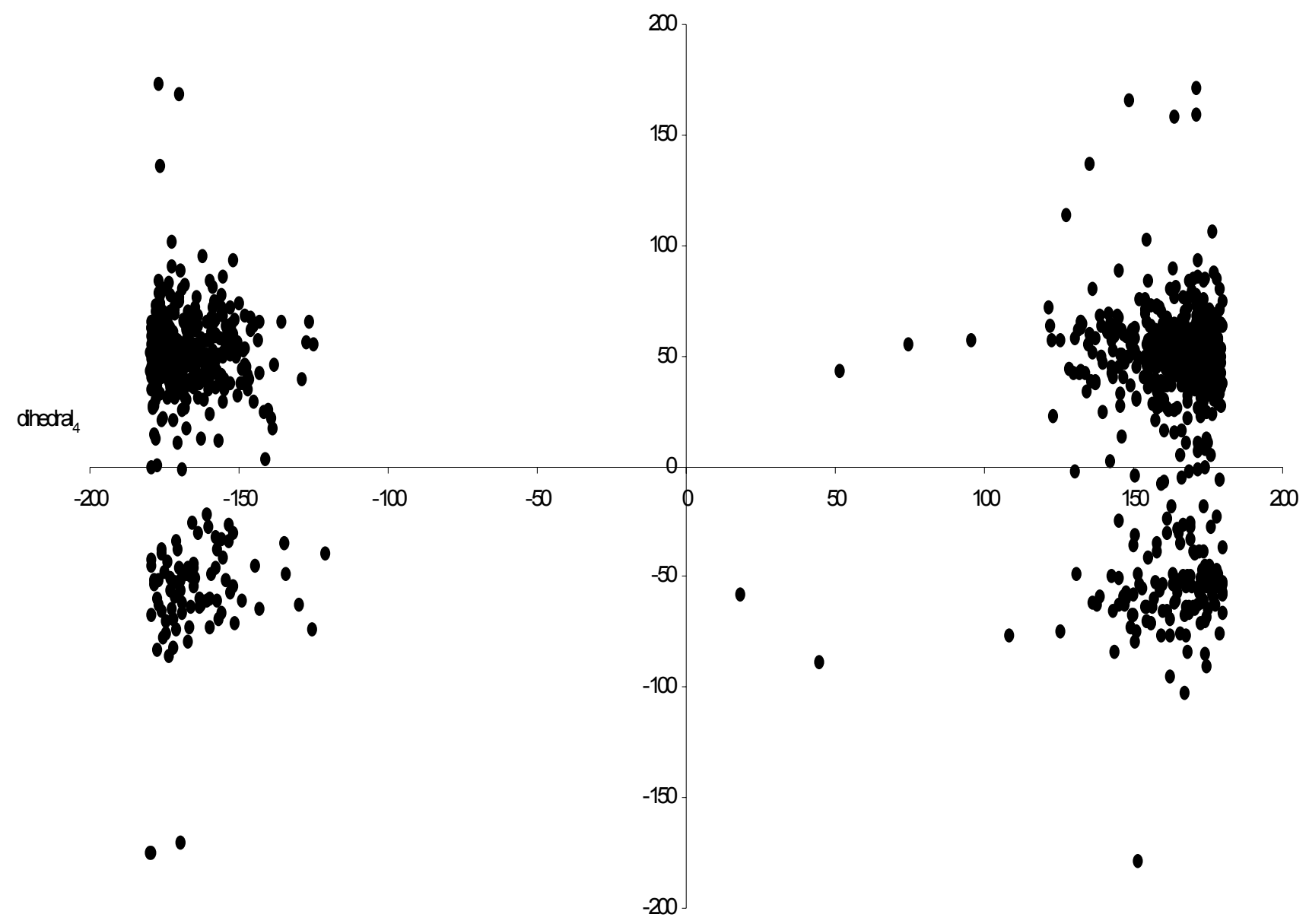

$w_{3}$

Figure S5. A plot of dihedral 4 vs. $\omega_{3}$ of 1,000 structures of 1 after 1-ns molecular dynamics at 600 K. 


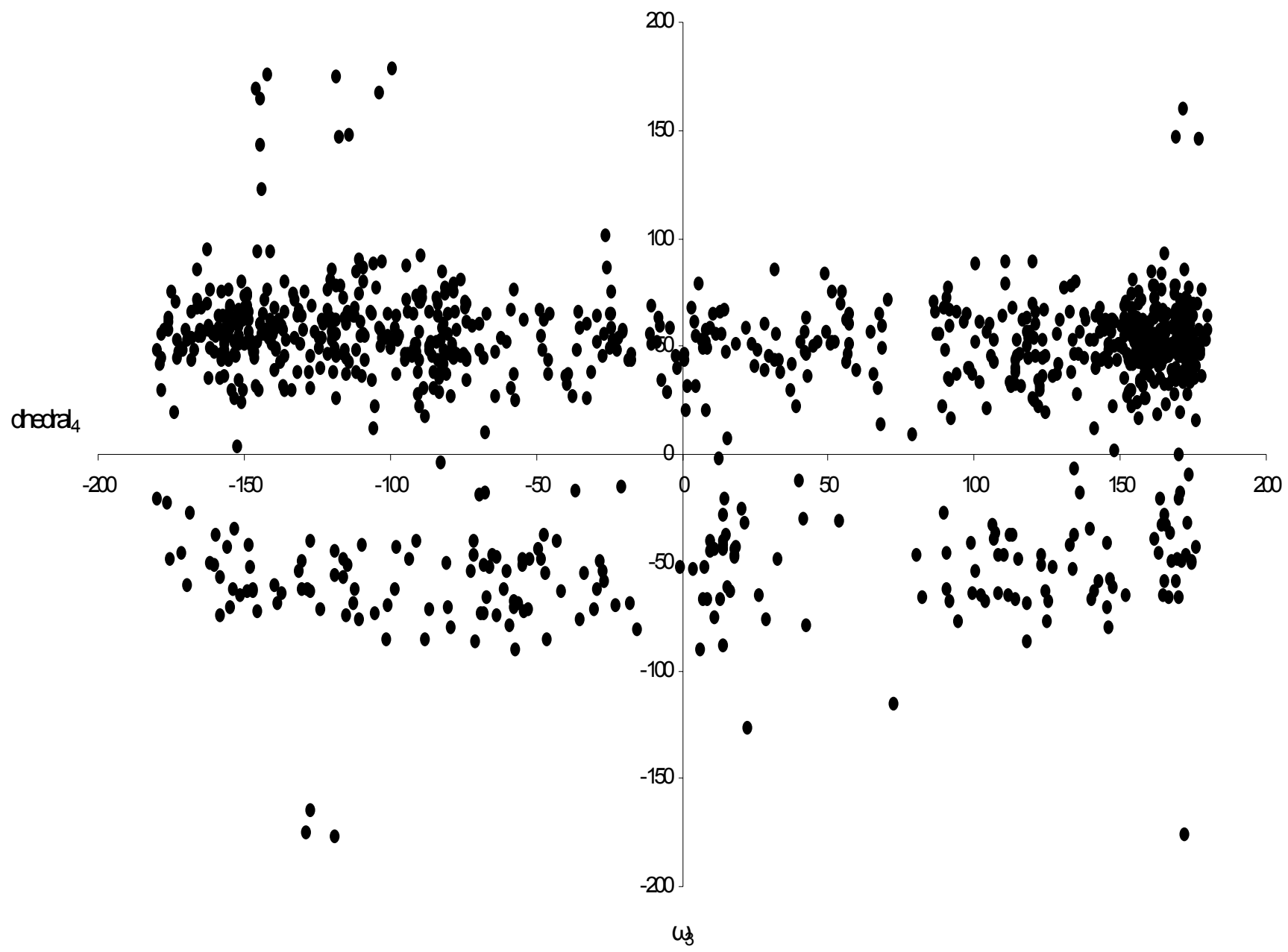

Figure S6. A plot of dihedral 4 vs. $\omega_{3}$ of 1,000 structures of 1 after minimization. 
Table S1. NMR-derived restraints of anthocyanin 2 in methanol- $d_{4}$.

\begin{tabular}{|c|c|c|}
\hline Proton-proton pairs & $\begin{array}{c}\text { Volume } \\
\text { measured } \\
\left(\times \mathbf{1 0}^{6}\right)\end{array}$ & $\begin{array}{l}\text { Strength of } \\
\text { interaction }^{a}\end{array}$ \\
\hline $\mathrm{H}-6^{\mathrm{IIIA}}-\mathrm{H}-6^{\mathrm{IIIB}}$ & 1.2 & $\mathrm{~S}$ \\
\hline $\mathrm{H}-7^{\mathrm{D}}-\mathrm{H}-2^{\mathrm{D}}$ & 0.39 & $\mathrm{~W}$ \\
\hline $\mathrm{H}-7^{\mathrm{D}}-\mathrm{H}-6^{\mathrm{D}}$ & $-{ }^{b}$ & $\mathrm{~W}$ \\
\hline $\mathrm{H}-7^{\mathrm{D}}-\mathrm{H}-8^{\mathrm{D}}$ & $-{ }^{b}$ & $\mathrm{~W}$ \\
\hline $\mathrm{H}-8^{\mathrm{D}}-\mathrm{H}-2^{\mathrm{D}}$ & $-{ }^{b}$ & $\mathrm{~W}$ \\
\hline $\mathrm{H}-8^{\mathrm{D}}-\mathrm{H}-6^{\mathrm{D}}$ & $-{ }^{b}$ & $\mathrm{~W}$ \\
\hline $\mathrm{H}-1^{\mathrm{I}}-\mathrm{H}-4$ & $-{ }^{b}$ & $\mathrm{~W}$ \\
\hline $\mathrm{H}-1^{\mathrm{I}}-\mathrm{H}-7^{\mathrm{D}}$ & $-{ }^{b}$ & $\mathrm{~W}$ \\
\hline $\mathrm{H}-1^{\mathrm{I}}-\mathrm{H}-3^{\mathrm{I}}$ & $-{ }^{b}$ & $\mathrm{~W}$ \\
\hline $\mathrm{H}-6^{\mathrm{IA}}-\mathrm{H}-6^{\mathrm{IB}}$ & $-{ }^{b}$ & $\mathrm{~W}$ \\
\hline
\end{tabular}




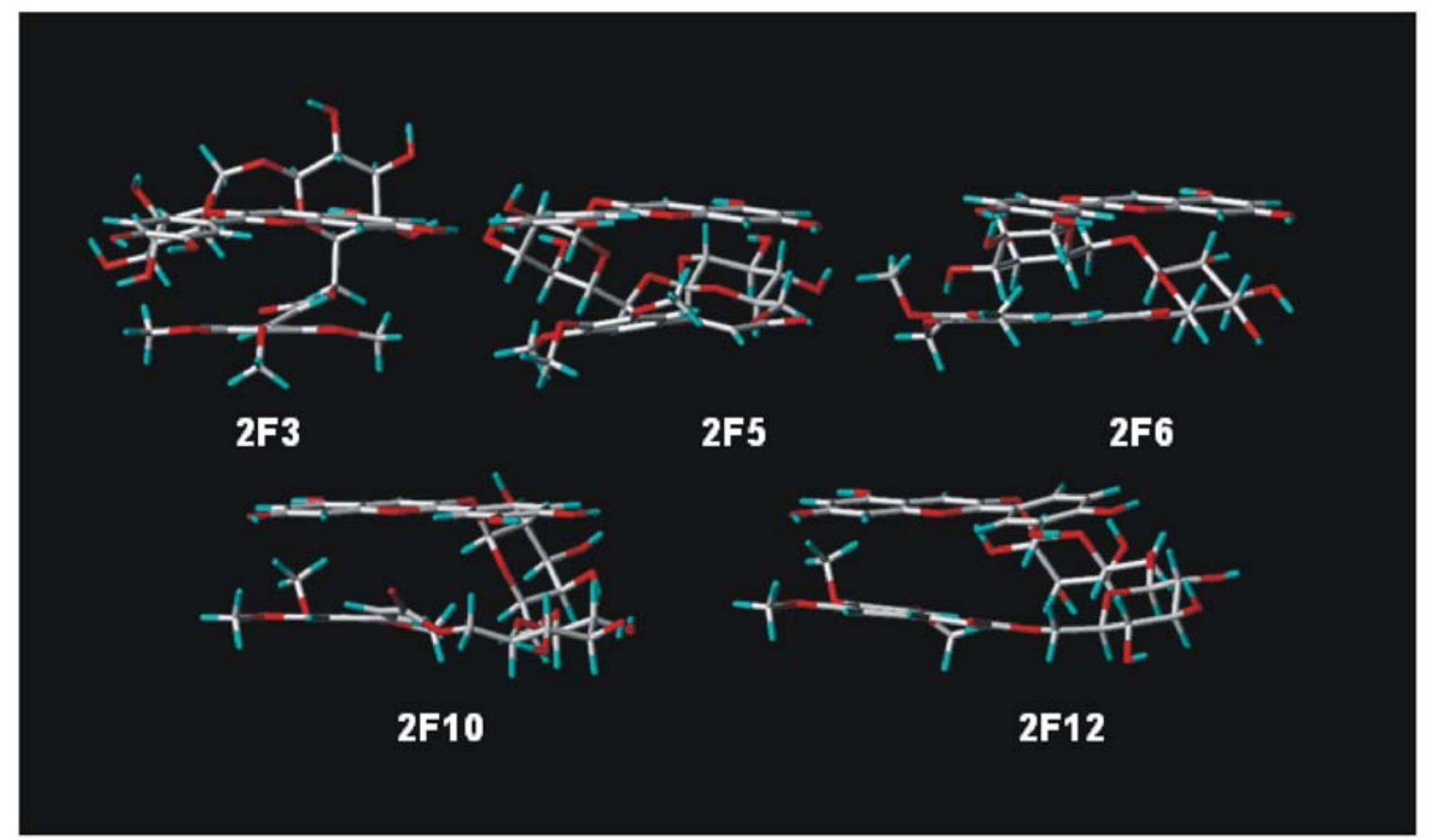

Figure S7. Conformational families of anthocyanin 2. 
Table S2. NMR-derived restraints of anthocyanin 3 in methanol- $d_{4}$.

\begin{tabular}{|c|c|c|}
\hline Proton-proton pair & $\begin{array}{c}\text { Volume } \\
\text { measured } \\
\left(\times 10^{6}\right)\end{array}$ & $\begin{array}{l}\text { Strength of } \\
\text { interaction }\end{array}$ \\
\hline H-6 $6^{\mathrm{IIIA}}-\mathrm{H}-6^{\mathrm{IIIB}}$ & 4.14 & $\mathrm{~S}$ \\
\hline $\mathrm{H}-6^{\mathrm{IA}}-\mathrm{H}-6^{\mathrm{IB}}$ & 2.72 & $\mathrm{~S}$ \\
\hline $\mathrm{H}-1^{\mathrm{II}}-\mathrm{H}-2^{\mathrm{I}}$ & 2.5 & M \\
\hline $\mathrm{H}-4^{\mathrm{I}}-\mathrm{H}-3^{\mathrm{I}}$ & 2.16 & M \\
\hline $\mathrm{H}-5^{\text {IIAeq }}-\mathrm{H}-5^{\text {IIBax }}$ & 2.03 & M \\
\hline $\mathrm{H}-2^{\mathrm{D}}-\mathrm{H}-8^{\mathrm{D}}$ & 1.81 & M \\
\hline $\mathrm{H}-6^{\mathrm{D}}-\mathrm{H}-8^{\mathrm{D}}$ & 1.81 & M \\
\hline $\mathrm{H}-2^{\mathrm{D}} / 6^{\mathrm{D}}-\mathrm{H} 3^{\mathrm{D}} / 5^{\mathrm{D}}$ & 1.79 & M \\
\hline $\mathrm{H}-1^{\mathrm{II}}-\mathrm{H}-5^{\mathrm{IIBax}}$ & 1.17 & $\mathrm{~W}$ \\
\hline $\mathrm{H}-7^{\mathrm{D}}-\mathrm{H}-2^{\mathrm{D}}$ & 1.11 & $\mathrm{~W}$ \\
\hline$H-7^{\mathrm{D}}-\mathrm{H}-6^{\mathrm{D}}$ & 1.11 & $\mathrm{~W}$ \\
\hline $\mathrm{H}-1^{\mathrm{II}}-\mathrm{H}-3^{\mathrm{I}}$ & 1.07 & $\mathrm{~W}$ \\
\hline $\mathrm{H}-4-\mathrm{H}-\mathrm{1}^{\mathrm{I}}$ & 1 & $\mathrm{~W}$ \\
\hline H-6 $6^{\mathrm{IIIB}}$ (Pro-S)-H-2 ${ }^{\mathrm{III}}$ & 0.751 & $\mathrm{~W}$ \\
\hline $\mathrm{H}-7^{\mathrm{D}}-\mathrm{H}-1^{\mathrm{I}}$ & 0.742 & $\mathrm{~W}$ \\
\hline H-6'-H-5' & 0.696 & $\mathrm{~W}$ \\
\hline $\begin{array}{l}\mathrm{H}-8^{\mathrm{D}}-\mathrm{H}-\mathrm{-}^{\mathrm{I}} \\
\mathrm{H}-1^{\mathrm{I}}-\mathrm{H}-3^{\mathrm{I}}\end{array}$ & $\begin{array}{l}0.445 \\
0.325\end{array}$ & $\begin{array}{l}\mathrm{W} \\
\mathrm{W}\end{array}$ \\
\hline H-6 ${ }^{\mathrm{IIIB}}$ (Pro-S)-H-4 & 0.141 & $\mathrm{~W}$ \\
\hline $\mathrm{H}-2^{\mathrm{I}}-\mathrm{H}-4^{\mathrm{I}}$ & 0.132 & $\mathrm{~W}$ \\
\hline $\mathrm{H}-7^{\mathrm{D}}-\mathrm{H}-8^{\mathrm{D}}$ & 0.131 & $\mathrm{~W}$ \\
\hline H- ${ }^{\mathrm{IA}}($ Pro-R $)-\mathrm{H}-4^{\mathrm{I}}$ & 0.0715 & $\mathrm{~W}$ \\
\hline
\end{tabular}

a. Strong interaction $(\mathrm{S})=1.7 \AA-2.5 \AA$; medium interaction $(\mathrm{M})=1.7 \AA-3.2 \AA$; weak interaction $(\mathrm{W})=1.7 \AA-5 \AA$. 


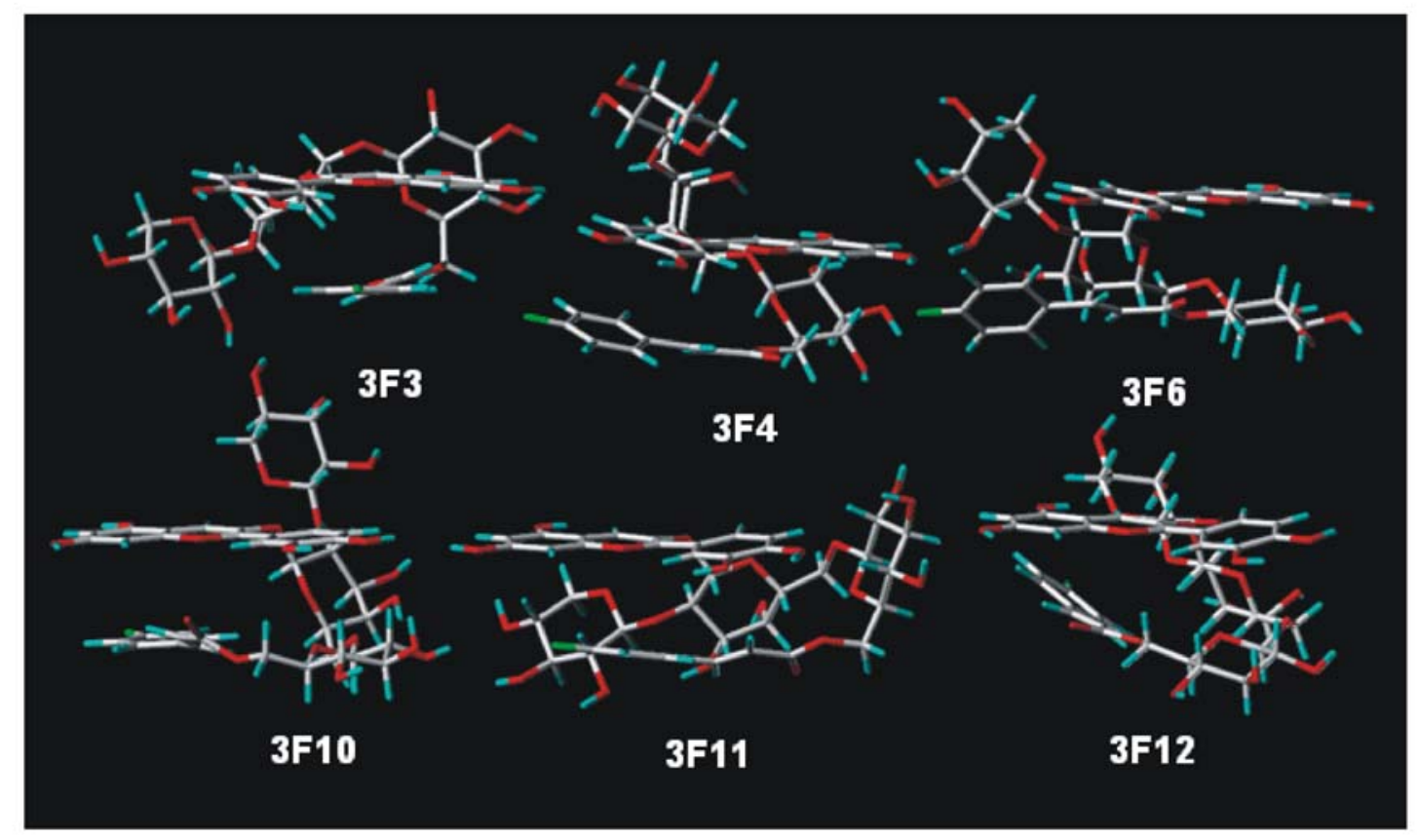

Figure S8. Conformational families of anthocyanin 3. 
Table S3. NMR-derived restraints of anthocyanin 4 in methanol- $d_{4}$.

\begin{tabular}{|c|c|c|}
\hline Proton-proton pairs & $\begin{array}{c}\text { Volume } \\
\text { measured } \\
\left(\times 10^{6}\right) \\
\end{array}$ & $\begin{array}{l}\text { Strength of } \\
\text { interaction }^{a}\end{array}$ \\
\hline H-6'-H-5' & 0.64 & $\mathbf{W}$ \\
\hline H-6 $^{\text {I }}$ (Pro R)-H-6 ${ }^{\text {I }}$ (Pro S) & 0.59 & $\mathbf{W}$ \\
\hline H-3 $-\mathbf{I}-\mathbf{H}-4^{\mathrm{I}}$ & 0.35 & $\mathbf{W}$ \\
\hline H-1 ${ }^{\mathrm{III}}-\mathbf{H}-3^{\mathrm{III}}$ & 0.34 & $\mathbf{W}$ \\
\hline H-1 ${ }^{\mathrm{III}}-\mathrm{H}-6^{\mathrm{I}}(\operatorname{Pro} \mathrm{S})^{b}$ & 0.32 & $\mathbf{W}$ \\
\hline H-1 ${ }^{\mathrm{III}}-\mathrm{H}-6^{\mathrm{I}}(\operatorname{Pro} \mathrm{R})^{b}$ & 0.32 & $\mathbf{W}$ \\
\hline H-6 ${ }^{\mathrm{D}}-\mathrm{OMe}$ & 0.31 & $\mathbf{W}$ \\
\hline H-2 ${ }^{\mathrm{D}}-\mathrm{OMe}$ & 0.31 & $\mathbf{W}$ \\
\hline H-5 $-\mathbf{I}-\mathbf{H}-4^{\mathrm{I}}$ & 0.27 & $\mathbf{W}$ \\
\hline 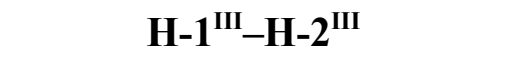 & 0.26 & $\mathbf{W}$ \\
\hline H-5 $-\mathbf{I}-\mathbf{H}-3^{I}$ & 0.25 & $\mathbf{W}$ \\
\hline H-1 $-\mathbf{I}^{\mathrm{I}}-\mathbf{6}^{\mathrm{D}}$ & 0.13 & $\mathbf{W}$ \\
\hline H-1 $-\mathbf{I}^{\mathrm{I}}-\mathbf{H}-2^{\mathrm{D}}$ & 0.13 & $\mathbf{W}$ \\
\hline H-4-H-1 ${ }^{\mathrm{I}}$ & 0.1 & $\mathbf{W}$ \\
\hline H-1 ${ }^{I}-\mathbf{H}-3^{I}$ & 0.096 & $\mathbf{W}$ \\
\hline H-5 $-\mathbf{H}^{\mathrm{I}} \mathbf{1}^{\mathrm{I}}$ & 0.081 & $\mathbf{W}$ \\
\hline H-1 ${ }^{\text {II }}-\mathbf{H}-3^{\text {I }}$ & 0.063 & $\mathbf{W}$ \\
\hline H-1 ${ }^{\text {II }}-\mathbf{H}-2^{\text {I }}$ & $-^{c}$ & $\mathbf{W}$ \\
\hline H-6 ${ }^{\mathrm{III}}$ (Pro R)-H-6 ${ }^{\mathrm{III}}$ (Pro S) & $-^{c}$ & $\mathbf{W}$ \\
\hline H-1 ${ }^{\mathrm{I}}-\mathbf{H}-2^{\mathrm{I}}$ & $-^{c}$ & $\mathbf{W}$ \\
\hline
\end{tabular}

a. Strong interaction $(\mathrm{S})=1.7 \AA-2.5 \AA$; medium interaction $(\mathrm{M})=1.7 \AA-3.2 \AA$; weak interaction $(\mathrm{W})=1.7 \AA-5 \AA$. b. Cannot determine whether $\mathrm{H}-6^{\mathrm{IB}}$ is Pro-R or Pro-S and so both were used to validate structures generated from modeling protocols. c. The ROE was confirmed by visual inspection of the spectrum, but its volume could not be measured accurately due to either $t_{1}$ noise or overlap of signals. All these are assigned as weak interactions. 


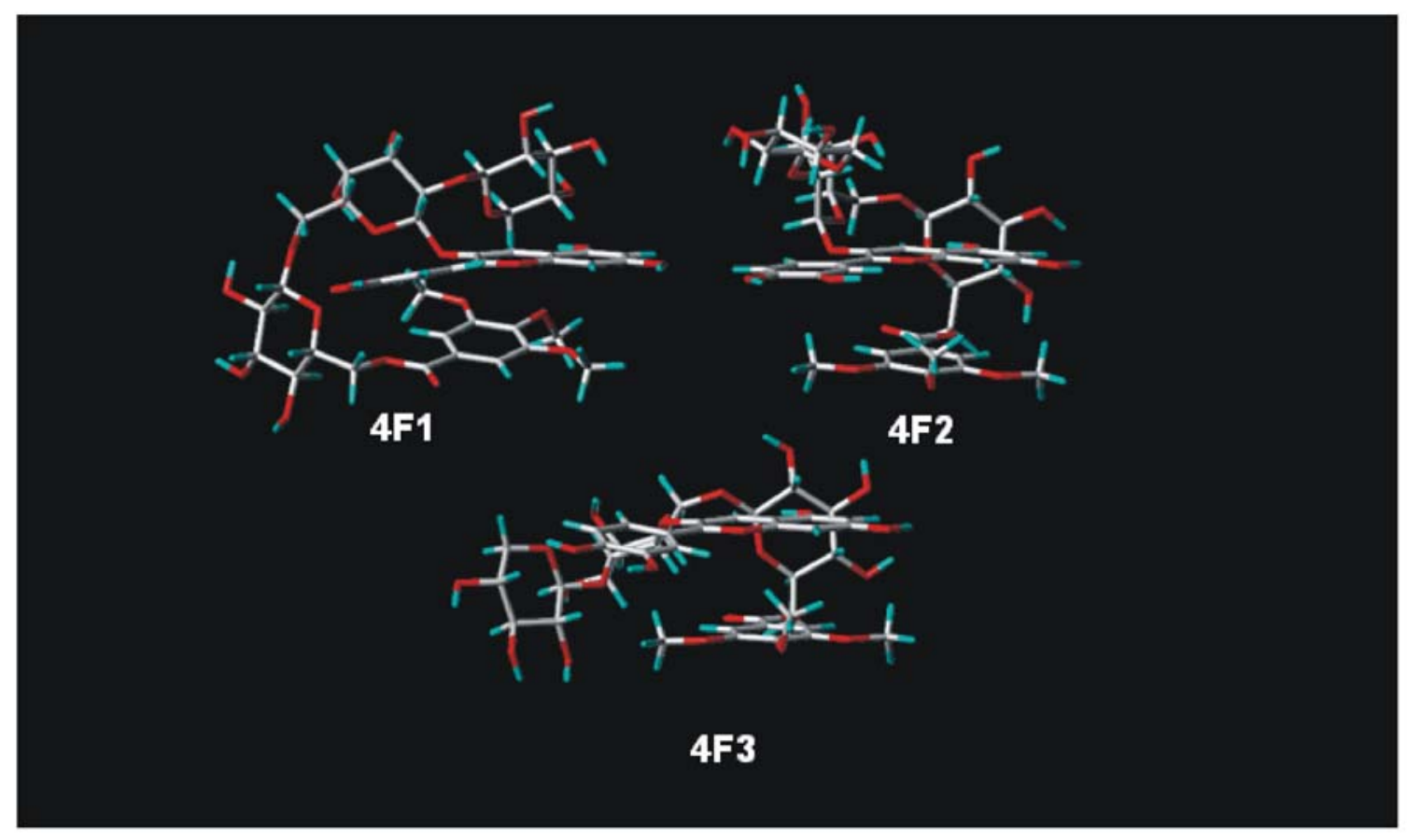

Figure S9. Some conformational families of anthocyanin 4 . 
Figure S10. The remaining conformations of families of anthocyanin 4.

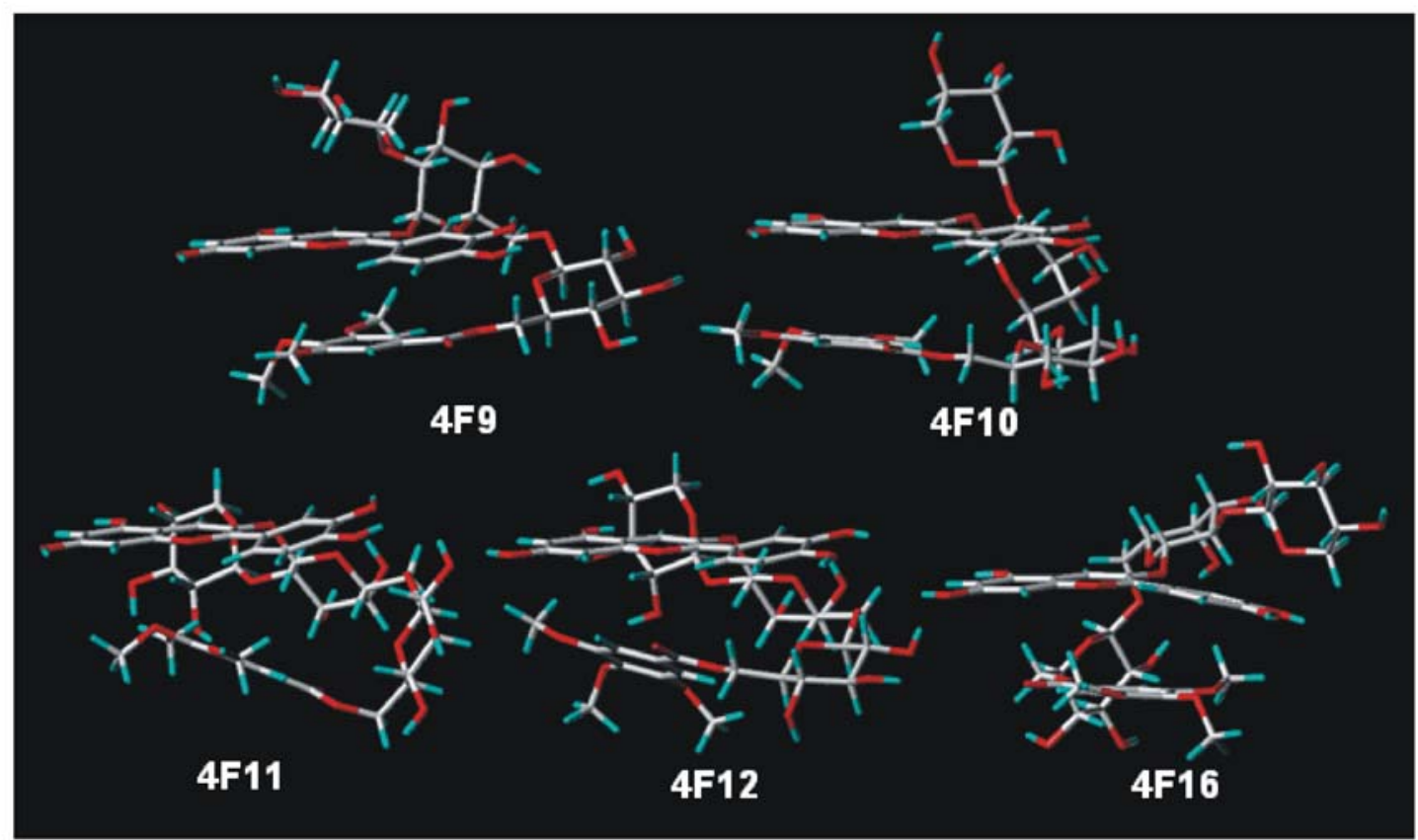


Table S4. NMR-derived restraints of anthocyanin 5 in methanol- $d_{4}$.

\begin{tabular}{|c|c|c|}
\hline Proton-proton pairs & $\begin{array}{c}\text { Volume } \\
\text { measured } \\
\left(\times 10^{6}\right) \\
\end{array}$ & Strength of interaction ${ }^{a}$ \\
\hline H-1 ${ }^{\mathrm{I}}-\mathrm{H}-6^{\mathrm{IB}}(\text { Pro-S })^{b}$ & 0.59 & $\mathrm{~W}$ \\
\hline H-1 $1^{\mathrm{I}}-\mathrm{H}-6^{\mathrm{IB}}(\text { Pro-R })^{b}$ & 0.59 & $\mathrm{~W}$ \\
\hline H-1 ${ }^{\mathrm{II}}-\mathrm{H}-2^{\mathrm{I}}$ & 0.47 & $\mathrm{~W}$ \\
\hline $\mathrm{H}-4-\mathrm{H}-1^{\mathrm{I}}$ & 0.45 & $\mathrm{~W}$ \\
\hline $\mathrm{H}-4-\mathrm{H}-2^{\mathrm{I}}$ & 0.21 & $\mathrm{~W}$ \\
\hline $\mathrm{H}-4^{\mathrm{I}-\mathrm{H}}-6^{\mathrm{IB}}(\text { Pro-R })^{b}$ & $-^{c}$ & $\mathrm{~W}$ \\
\hline $\mathrm{H}-4^{\mathrm{I}}-\mathrm{H}-6^{\mathrm{IB}}(\text { Pro-S })^{b}$ & $-^{c}$ & $\mathrm{~W}$ \\
\hline $\mathrm{H}-2^{\mathrm{III}}-\mathrm{H}-6^{\mathrm{IIIA}}(\text { Pro-R })^{b}$ & $-^{c}$ & W \\
\hline H-2 ${ }^{\mathrm{III}}-\mathrm{H}-6^{\mathrm{IIIA}}(\text { Pro-S })^{b}$ & $-^{c}$ & $\mathrm{~W}$ \\
\hline $\mathrm{H}-2^{\mathrm{III}}-\mathrm{H}-2^{\mathrm{I}}$ & $-^{c}$ & $\mathrm{~W}$ \\
\hline $\mathrm{H}-5^{\mathrm{IIBax}}-\mathrm{H}-4^{\mathrm{II}}$ & $-^{c}$ & $\mathrm{~W}$ \\
\hline $\mathrm{H}-1^{\mathrm{III}}-\mathrm{H}-2^{\mathrm{III}}$ & $-^{c}$ & $\mathrm{~W}$ \\
\hline $\mathrm{H}-5^{\mathrm{IIBax}}-\mathrm{H}-2^{\mathrm{II}}$ & $-^{c}$ & $\mathrm{~W}$ \\
\hline H-6 $6^{\text {IIIA }}(\text { Pro-S })^{b}-\mathrm{H}-2^{\mathrm{I}}$ & $-^{c}$ & $\mathrm{~W}$ \\
\hline H-6 ${ }^{\text {IIIA }}(\text { Pro- } \mathrm{R})^{b}-\mathrm{H}-2^{\mathrm{I}}$ & $-^{c}$ & W \\
\hline
\end{tabular}

a. Strong interaction $(\mathrm{S})=1.7 \AA-2.5 \AA$; medium interaction $(\mathrm{M})=1.7 \AA-3.2 \AA$; weak interaction $(\mathrm{W})=1.7 \AA-5 \AA$. b. Cannot determine whether $\mathrm{H}-6^{\mathrm{IB}}$ is Pro-R or Pro-S and so both were used to validate structures generated from modeling protocols. c. The ROE that was confirmed by visual inspection of the spectrum, but its volume could not be measured accurately due to either $t_{1}$ noise or overlap of signals. All these were assigned as weak interactions. 


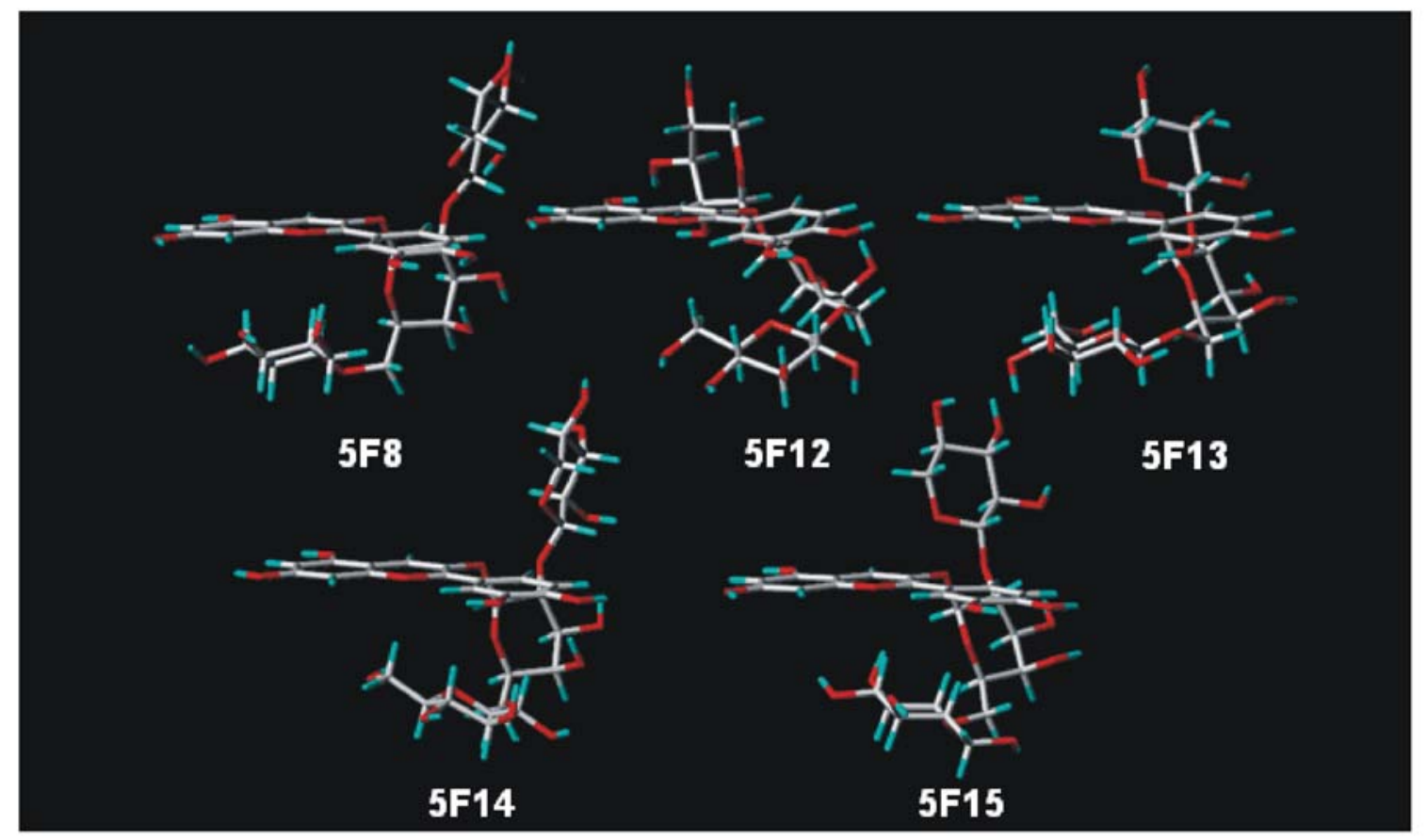

Figure S11. Conformational families of anthocyanin 5. 